\title{
THE STRUCTURE OF GLIOVIRIN, A NEW ANTIBIOTIC FROM GLIOCLADIUM VIRENS
}

\author{
Robert D. Stipanovic and Charles R. Howell \\ U. S. Dept. of Agriculture, Agricultural Research Service \\ National Cotton Pathology Research Laboratory \\ P. O. Drawer JF \\ College Station, Texas 77841, U.S.A.
}

(Received for publication June 28, 1982)

\begin{abstract}
Gliovirin, a new antibiotic active against Pythium ultimum, has been isolated from Gliocladium virens. The structure of gliovirin was determined by NMR, mass spectral, and X-ray crystallographic analyses. Gliovirin may be derived from L,L-phenylalanine anhydride, which was also isolated from $G$. virens.
\end{abstract}

In an earlier study, we reported the presence of the antibiotic, heptelidic acid, in the fungus, Gliocladium virens ${ }^{1}$. From this same organism we have isolated two additional compounds, one of which is selectively active against members of the Oomycetes ${ }^{2}$. We call this compound gliovirin (1). The second compound, L,L-phenylalanine anhydride (9), may be the biosynthetic precursor to gliovirin.

Gliovirin had a mp of $247 \sim 249^{\circ} \mathrm{C}$. It gave a parent ion in the mass spectrum at $480 \mathrm{~m} / \mathrm{z}(13 \%)$. The intensity of the $\mathbf{P}+1(3.5 \%)$ peak and the $\mathbf{P}+2(2.3 \%)$ peak and the fragment ions at $\mathrm{m} / z 448(4 \%$, $\mathrm{P}-32)$, and $\mathrm{m} / \mathrm{z} 416(17 \%, \mathrm{P}-64)$ indicated the possibility of a disulfide group in the molecule. On silica gel plates, gliovirin gave a positive reaction when sprayed with $\mathrm{AgNO}_{3}$. Epipolythiodioxopiperazines give this reaction ${ }^{3)}$. Disulfides, such as gliotoxin and related compounds, have been isolated from other Gliocladium species ${ }^{4,5)}$. However, unlike gliotoxin which gives a base peak at $\mathrm{P}-64^{6)}$, gliovirin tends to lose both 64 and 65 amu to give peaks at $m / z 416(17 \%)$ and $m / z 415(25 \%)$, respectively. The intense peak at $m / z 388(79 \%)$ may arise by the loss of CO from the $m / z 415$ ion. This is a common fragmentation of diketopiperazines, but not of gliotoxin which contains an 2,5-epidithio-3,6-diketo group. A high resolution mass spectrum was not obtained because the sample rapidly decomposed at the temperature $\left(360^{\circ} \mathrm{C}\right)$ required to volatilize it. However, an elemental analysis and the low resolution mass spectral data provided the formula $\mathrm{C}_{20} \mathrm{H}_{20} \mathrm{~N}_{2} \mathrm{O}_{8} \mathrm{~S}_{2}$, indicating 12 degrees of unsaturation.

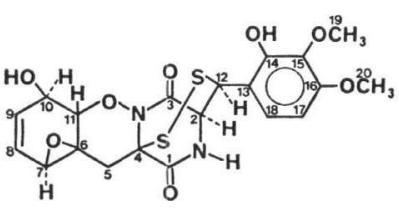

(1)

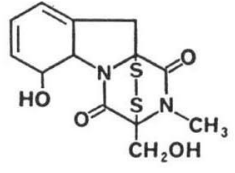

(2)
Table $1 .{ }^{13} \mathrm{C}$ Chemical shift assignments for gliovirin in $\left(\mathrm{CD}_{3}\right)_{2} \mathrm{SO}^{*}$.

\begin{tabular}{cc|cc}
\hline Carbon & $\delta$ & Carbon & $\delta$ \\
\hline$>\mathrm{C}=\mathrm{O}$ & $166.1(\mathrm{~s})$ & 11 & $86.2(\mathrm{~d})$ \\
$>\mathrm{C}=\mathrm{O}$ & $163.1(\mathrm{~s})$ & 12 & $43.9(\mathrm{~d})$ \\
2 & $52.7(\mathrm{~d})$ & 13 & $116.0(\mathrm{~s})$ \\
4 & $70.3(\mathrm{~s})$ & 14 & $148.1(\mathrm{~s})$ \\
5 & $33.4(\mathrm{t})$ & 15 & $136.2(\mathrm{~s})$ \\
6 & $58.0(\mathrm{~s})$ & 16 & $153.5(\mathrm{~s})$ \\
7 & $60.1(\mathrm{~d})$ & 17 & $103.7(\mathrm{~d})$ \\
8 & $122.7(\mathrm{~d})$ & 18 & $123.1(\mathrm{~d})$ \\
9 & $138.2(\mathrm{~d})$ & 19 & $60.4(\mathrm{q})$ \\
10 & $66.0(\mathrm{~d})$ & 20 & $55.9(\mathrm{q})$ \\
\hline
\end{tabular}

* Chemical shifts are in ppm down field from TMS using the central resonance of $\left(\mathrm{CD}_{3}\right)_{2} \mathrm{SO}(\delta$ 39.6) as an internal reference; $(\mathrm{s})=$ singlet, $(\mathrm{d})=$ doublet, $(\mathrm{t})=$ triplet, $(\mathrm{q})=$ quartet. 
Two carbonyl groups, with chemical shifts characteristic of amides, were observed in the ${ }^{13} \mathrm{C} \mathrm{NMR}$ spectrum ( $\delta$ 166.1 and 163.1) (Table 1). The ${ }^{13} \mathrm{C}$ NMR indicated four C-C double bonds. Four of these carbons were singly protonated and four were completely substituted. The UV spectrum [ $\lambda_{\max }^{\mathrm{EtoH}}$

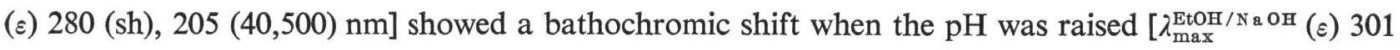
$(5,400) \mathrm{nm}]$, indicating the presence of a phenol. The coupling $(J=8.9 \mathrm{~Hz})$ between the two aromatic protons ( $\delta 7.41$ and 6.55 ) (Table 2$)$ indicated that they were on adjacent carbons. Two methoxyl groups were evident in both the ${ }^{1} \mathrm{H}$ NMR $[\delta 3.77(3 \mathrm{H}, \mathrm{s})$ and $3.65(3 \mathrm{H}, \mathrm{s})]$ and ${ }^{13} \mathrm{C}$ NMR $[\delta 60.9$ (q) and 55.9 (q)] spectra. The three aromatic carbons at $\delta 153.5$ (s), 148.1 (s) and 136.2 (s) appeared to have oxygen substituents. By comparison with model compounds such as 1,2,3-trimethoxybenzene (3) and 1-hydroxy2,3-dimethoxybenzene (4), it was evident that these oxygenated carbons were adjacent to one another. Thus it was possible to make the chemical shift assignments and propose partial structure (5). In structure $\mathbf{5}$ it is assumed that the aromatic ring is derived from phenyalanine, and it is, therefore, connected by one carbon to the diketopiperazine ring. The disulfide group is assumed to bridge, in some manner, the diketopiperazine ring.

An isolated C-C double bond is also present in the molecule. The vinyl protons appearing at $\delta$ 5.87 and 5.76 and the proton at $\delta 3.10$ make up an $\mathrm{ABX}$ system with $J_{\mathrm{AB}}=10.8 \mathrm{~Hz}$ (cis-substituted olefin), $J_{\mathrm{AX}}=0$ and $J_{\mathrm{BX}}=2.0 \mathrm{~Hz}$. This provides partial structure 6 . Partial structures 5 and $\mathbf{6}$ with the disulfide bridge account for $\mathrm{C}_{13} \mathrm{H}_{8} \mathrm{~N}_{2} \mathrm{O}_{5} \mathrm{~S}_{2}$; unaccounted for are $\mathrm{C}_{7} \mathrm{H}_{11} \mathrm{O}_{3}$ and 3-degrees of unsaturation which must be in the form of rings.

Although additional information could be gleaned from the NMR spectra, it was not possible to unambiguously determine the remainder of the molecule, the attachment of the disulfide bridge, or the absolute configuration. A sample was therefore submitted for single crystal X-ray analysis. A crystal suitable for X-ray analysis was obtained from acetone. Gliovirin crystallized with one molecule of water. A perspective view of gliovirin is given in Fig. 1 .

Fig. 1. Crystal structure of gliovirin.

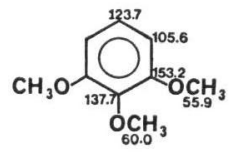

(3)

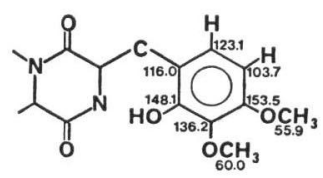

(5)

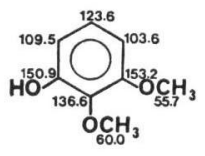

(4)

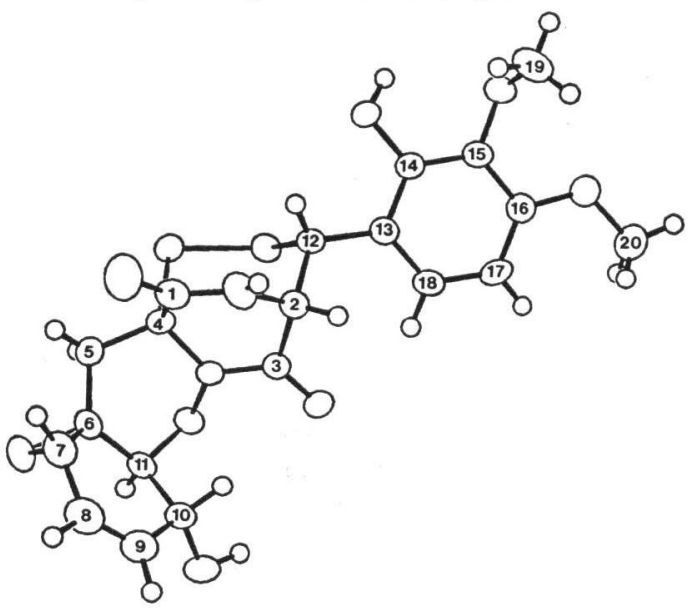

X-Ray Crystallography

Crystal data: $\mathrm{C}_{20} \mathrm{H}_{20} \mathrm{~N}_{2} \mathrm{O}_{9} \mathrm{~S}_{2} \cdot \mathrm{H}_{2} \mathrm{O}$

F. W. 498.53 , crystal dimensions $0.15 \times 0.15 \times 0.20 \mathrm{~mm}$.

Monoclinic, $a=9.312$ (1), $b=9.399$ (3), $c=12.370$ (2) $\AA$.

$\mathrm{B}=91.41(1)^{\circ}, \mathrm{V}=1082.4 \mathrm{~A}^{3}$, space group $\mathrm{P} 2$.

$\mathrm{Z}=2, \mathrm{p}=1.53 \mathrm{~g} / \mathrm{cm}^{3}, \mathrm{CuK} \alpha$ radiation, $\lambda=1.54184 \AA, \mu=26.7 \mathrm{~cm}^{-1}$.

Temperature $=23 \pm 1^{\circ} \mathrm{C}$. 
Intensity data were measured on an Enraf-Nonius CAD4 diffractometer equipped with a graphite crystal. A total of 2468 reflections were collected, of which 2374 were unique and not systematically absent. The structure was solved by direct methods. Using 220 reflections (minimum E of 1.54) and 1970 relationships, a total of 32 phase sets were produced. A total of 8 atoms were located from an E-map prepared from the phase set with probability statistics: absolute figure of merit $=1.34$, residual $=$ 27.80 and psi zero $=2.140$. The remaining atoms were located in succeeding difference Fourier synthesis. Hydrogen atoms were located and their position and isothermal parameters were refined. A refinement of the other enantiomorph showed clearly by a difference in R2 of 0.005 that the present enantiomorph is the correct one.

Gliovirin is probably derived from two molecules of phenylalanine. The disulfide group does not bridge directly across the diketopiperazine ring as in antibiotics such as gliotoxin (2). Gliovirin also contains an alkoxyamino group.

Using the X-ray crystal structure, both the ${ }^{1} \mathrm{H}$ and ${ }^{13} \mathrm{C}$ NMR spectra could be completely interpreted. The vinyl protons at $\delta 5.87$ and 5.76 are strongly coupled to each other and each is weakly coupled to the protons at $\delta 3.10(2.0 \mathrm{~Hz})$, and $\delta 4.27(<1 \mathrm{~Hz})$, respectively. The X-ray analysis shows that the torsional angle between $\mathrm{H}-\mathrm{C}(8)-\mathrm{C}(7)-\mathrm{H}$ is $37.0^{\circ}$ and therefore these protons will show coupling; but the torsional angle between $\mathrm{H}-\mathrm{C}(9)-\mathrm{C}(10)-\mathrm{H}$ is $88.9^{\circ}$, and therefore these protons will be only weakly coupled. This allows assignment of: $\mathrm{C}(8)-\mathrm{H} \delta 5.87 ; \mathrm{C}(7)-\mathrm{H} \delta 3.10, \mathrm{C}(9)-\mathrm{H} \delta 5.76$, and $\mathrm{C}(11)-\mathrm{H} \delta 4.27$. Double irradiation experiments confirmed these assignments, and showed that the proton at $\delta 4.27$ also was coupled to the broad singlet at $\delta$ 5.50. Since this peak is exchanged with $\mathrm{D}_{2} \mathrm{O}$ it is assigned to the $-\mathrm{OH}$ group at $\mathrm{C}(10)$. This unusually strong coupling through the $-\mathrm{OH}$ group is caused by a slow exchange rate. Double irradiation experiments showed this slow exchange rate was caused by intermolecular hydrogen bonding with the $\mathrm{N}-\mathrm{H}$ proton $(\delta 9.03)$. This proton also showed strong coupling to the multiplet at $\delta 4.45$; and this peak is assigned to $\mathrm{C}(2)-\mathrm{H}$. Similarly this coupling must result from the slow exchange rate. $\mathrm{C}(10)-\mathrm{H}(\delta 4.27)$ coincidentally overlaps with the $\mathrm{C}(11)-\mathrm{H}$ and they appear as a broad singlet. The $\mathrm{C}(12)$ and $\mathrm{C}(2)$ protons appear as an $\mathrm{AB}$ multiplet at $\delta 4.43$ and 4.45 , respectively. The protons on $\mathrm{C}(5)$ are coupled only to each other and appear as a broad singlet at $\delta 2.57$. The phenol proton is found as a sharp $\mathrm{D}_{2} \mathrm{O}$ exchanged singlet at $\delta 9.56$.

Unassigned peaks in the ${ }^{13} \mathrm{C}$ NMR spectrum were identified by selective proton decoupling experiments, completely proton-decoupled experiments, and comparison with such model compounds as 3cyclohexenol (7) and 3-cyclohexeneepoxide (8). The assignments are listed in Table 1.

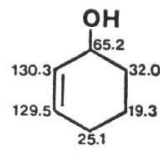

(7)

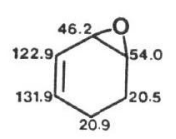

(8)

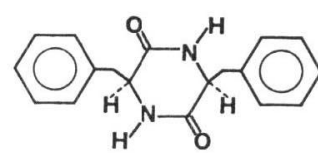

(9)

The second compound which precipitated from the crude acetone solutions of gliovirin had a mp of $303 \sim 308^{\circ} \mathrm{C}$ (methanol) with sublimation. It gave a parent peak in the mass spectrum at $\mathrm{m} / \mathrm{z} 294$, and a base peak at $m / z$ 91. Its ${ }^{13} \mathrm{C}$ NMR spectrum indicated it was phenylalanine anhydride. It had a rotation of $[\alpha]_{D}^{]^{5}}-167^{\circ},[\alpha]_{548}^{55}-200^{\circ}(c 0.054$, pyridine). Literature reports for L, L-phenylalanine anhydride are $[\alpha]_{\mathrm{D}}^{25}-242^{\circ},[\alpha]_{548}^{25}-270^{\circ}(c 0.059 \text {, pyridine })^{7)}$, and $\left.[\alpha]_{548}^{25}-267 \pm 3^{\circ}(c 0.132 \text {, pyridine })^{8}\right)$, and $\mathrm{mp}$ 
$326^{\circ} \mathrm{C}^{8)}$ and $311 \sim 312^{\circ} \mathrm{C}$ (methanol) $)^{7)}$; for D,D-phenylalanine anhydride $[\alpha]_{D}^{25}+107^{\circ}$ (c 0.223 , ethanol) and $\mathrm{mp} 315 \sim 316^{\circ} \mathrm{C}^{9)}$ are reported.

L,L-, D,D- And D,L-phenylalanine anhydride were prepared by the general procedure of KIRBY et al ${ }^{10)}$. L,L-Phenylalanine anhydride (9) had $\mathrm{mp} 317 \sim 320^{\circ} \mathrm{C}$ with sublimation (methanol) and rotation of $[\alpha]_{D}^{25}$ $-220^{\circ},[\alpha]_{548}^{25}-266^{\circ}\left(c 0.089\right.$, pyridine). D,L-Phenylalanine anhydride rapidly sublimed at $256^{\circ} \mathrm{C}$ and did not melt. D,D-Phenylalanine anhydride had $\mathrm{mp} 326 \sim 328^{\circ} \mathrm{C}$ with sublimation (methanol). The ${ }^{13} \mathrm{C}$ NMR spectrum of the L,L-enantiomer was distinctly different from that of the D,L-enantiomer, but was virtually identical to that of the natural product (see Experimental Section). A careful examination of the ${ }^{13} \mathrm{C}$ NMR spectrum of the natural compound indicated the presence of trace impurities that were not removed by recrystallization. These impurities probably account for the discrepancies in melting point and rotation indicated above.

Since L,L-phenylalanine anhydride was isolated from culture media, it may act as the biosynthetic precursor to gliovirin. However, feeding D- and L-phenylalanine, the L,L-, the D,L- and the D,D-enantiomers of phenylalanine anhydride to separate shake cultures of $G$. virens did not increase the concentration of gliovirin. Many factors govern the uptake and utilization of different substrates, thus the biosynthetic origin of gliovirin remains uncertain.

\section{Experimental}

Melting points were determined on a Kofler hot-stage microscope and are uncorrected. Optical rotations were determined on a Perkin-Elmer Model 241 polarimeter. Mass spectra were measured at low resolution on a Varian MAT-CH7 spectrometer. The ${ }^{1} \mathrm{H}$ NMR and ${ }^{13} \mathrm{C}$ NMR spectra were recorded on either a JEOL FX-90Q or a Varian XL-200 using $\left(\mathrm{CD}_{3}\right)_{2} \mathrm{SO}$ as solvent. Elemental analyses were determined by Galbraith Laboratories. The single crystal X-ray structure determination was performed by Molecular Structure Corporation. Complete X-ray data is deposited with the Cambridge Crystallographic Data Center. Cultural techniques used to grow G. virens and the procedures used to isolate and purify gliovirin and L,L-phenylalanine anhydride have been described $^{2)}$.

Gliovirin (1)

MS (Probe $\left.360^{\circ} \mathrm{C}\right) \mathrm{m} / \mathrm{z}(\%): 482(2, \mathrm{M}+2), 481(3.5 \mathrm{M}+1), 480$ (13.4, M), $416(17), 415(25), 389(17)$, 388 (79), 290 (20), 270 (20), 263 (14), 248 (28), 247 (19), 232 (13), 231 (34), 230 (12), 222 (20), 221 (100), 207 (14), 206 (56), 200 (11), 199 (71), 198 (27), 197 (26), 194 (13), 193 (12), 192 (28), 179 (19), 178 (84), 177 (12). $[\alpha]_{D}^{25}-97^{\circ}(c 0.035$, methanol).

Anal. Calcd. for $\mathrm{C}_{20} \mathrm{H}_{20} \mathrm{~N}_{2} \mathrm{O}_{8} \mathrm{~S}_{2}$ : C 49.99, $\mathrm{H} 4.20, \mathrm{~N} 5.83, \mathrm{~S} 13.35$.

Found:

C $49.63, \mathrm{H} 4.44, \mathrm{~N} 5.59, \mathrm{~S} 13.39$.

L,L-Phenylalanine Anhydride (9)

${ }^{13} \mathrm{C} \mathrm{NMR}\left[\left(\mathrm{CD}_{3}\right)_{2} \mathrm{SO}, 50^{\circ} \mathrm{C}\right] \delta 166.1(\mathrm{C}=\mathrm{O}), 136.5(\mathrm{C}-4), 129.7,128.1(\mathrm{C}-5, \mathrm{C}-6), 126.3(\mathrm{C}-7), 55.4$ (C-2), 39.3 (C-3). MS (Probe 225 C) m/z (\%): 294 (10, M), 175 (16), 120 (12), 93 (13), 91 (100), 65 (17). D,L-Phenylalanine Anhydride

${ }^{13} \mathrm{C} \mathrm{NMR}\left[\left(\mathrm{CD}_{3}\right)_{2} \mathrm{SO}, 60^{\circ} \mathrm{C}\right] \delta 166.7(\mathrm{C}=\mathrm{O}), 135.9(\mathrm{C}-4), 129.8,127.7(\mathrm{C}-5, \mathrm{C}-6), 126.4(\mathrm{C}-7), 54.6$ (C-2), 37.8 (C-3).

\section{Acknowledgements}

We thank: Robin Ford, JAN Cornish and Lucile Young for excellent technical assistance; Steve Silber for $200 \mathrm{MHz}$ NMR spectra; MARIE OSBORNE for assistance with chemical nomenclature; and HowARD Williams for helpful discussions.

Mention of firm names or trade products does not imply that they are endorsed by the U. S. Department of Agriculture over other firms or similar products not mentioned. 


\section{References}

1) Stipanovic, R. D. \& C. R. Howell: The X-ray crystal structure determination, and biosynthetic studies of the antibiotic, heptelidic acid. Tetrahedron. in press

2) Howell, C. R. \& R. D. Stipanovic: Gliovirin, a new antibiotic from Gliocladium virens, and its role in the biological control of Pythium ultimum. Canad. J. Microbiol. in press.

3) Rahman, R.; S. SAFE \& A. TAYlor: Separation of polythiadioxopiperazine antibiotics by thin-layer chromatography. J. Chromatogr. 53: 592 594, 1970

4) Kirby, G. W.; D. J. Robins, M. A. Sefton \& R. R. Talekar: Biosynthesis of bisdethiobis(methylthio)gliotoxin, a new metabolite of Gliocladium deliquescens. J. Chem. Soc., Perkin I 1980: 119 121, 1980

5) Hanson, J. R. \& M. A. O'Leary: New piperazinedione metabolites of Gliocladium deliquescens. J. Chem. Soc., Perkin I 1981: 218 220, 1981

6) Okutani, K.: Gliotoxin produced by a strain of Aspergillus isolated from marine mud. Bull. Japan. Soc. Scien. Fish. 48: 995 1000, 1977

7) Brown, R.; C. Kelley \& S. E. Wiberley: The production of 3-benzylidene-6-isobutylidene-2,5-dioxopiperazine, 3,6-dibenzylidene-2,5-dioxopiperazine, and 3,6-dibenzyl-2,5-dioxopiperazine by a variant of Streptomyces noursei. J. Org. Chem. 30: 277 280, 1965

8) Brinkenshaw, J. H. \& Y. S. Mohammed: Studies in the biochemistry of microorganisms. III. The production of L-phenylalanine anhydride (cis-L-3,6-dibenzyl-2,5-dioxopiperazine) by Penicillium nigricans (Bainier) Thom. Biochem. J. 85: 523 527, 1962

9) VeJdlek, Z. J.: Stereoisomeric dipeptides of phenylalanine. Collect. Czech. Chem. Comm. 15: 926 936, 1950

10) Kirby, G. W.; G. L. Patrick \& D. J. Roberts: Cyclo-(L-phenylalanyl-L-selyl) as an intermediate in the biosynthesis of gliotoxin. J. Chem. Soc., Perkin I 1978: 1336 1338, 1978 